\title{
Riemann Zeta Based Surge Modelling of Continuous Real Functions in Electrical Circuits
}

\author{
Binesh Thankappan \\ Govt. Model Engineering College-APJ Abdul Kalam Technological University, Ernakulam, India - \\ 682021 \\ Email: binesht@mec.ac.in
}

Received: June 24, 2021. Revised: December 16, 2021. Accepted: January 14, 2022. Published: January 15, 2022.

\begin{abstract}
Riemann zeta is defined as a function of a complex variable that analytically continues the sum of the Dirichlet series, when the real part is greater than unity. In this paper, the Riemann zeta associated with the finite energy possessed by a $2 \mathrm{~mm}$ radius, free falling water droplet, crashing into a plane is considered. A modified zeta function is proposed which is incorporated to the spherical coordinates and real analysis has been performed. Through real analytic continuation, the single point of contact of the drop at the instant of touching the plane is analyzed. The zeta function is extracted at the point of destruction of the drop, where it defines a unique real function. A special property is assumed for some continuous functions, where the function's first derivative and first integral combine together to a nullity at all points. Approximate reverse synthesis of such a function resulted in a special waveform named the dying-surge. Extending the proposed concept to general continuous real functions resulted in the synthesis of the corresponding function's Dying-surge model. The Riemann zeta function associated with the water droplet can also be modeled as a dying-surge. The Dying- surge model corresponds to an electrical squeezing or compression of a waveform, which was originally defined over infinite arguments, squeezed to a finite number of values for arguments placed very close together with defined final and penultimate values. Synthesized results using simulation software are also presented, along with the analysis. The presence of surges in electrical circuits will correspond to electrical compression of some unknown continuous, real current or voltage function and the method can be used to estimate the original unknown function.
\end{abstract}

Keywords : Riemann zeta, Continuous real functions, Spherical coordinates, Surge waveform, Dying -surge model, Electrical squeezing.

\section{INTRODUCTION}

In this paper the Riemann zeta associated with the finite energy possessed by a $2 \mathrm{~mm}$ radius free falling water droplet just collapsing on a surface is analyzed. The droplet, assuming a spherical geometry, falling through a distance under the influence of gravity, is crashing onto a horizontal plane. The initial single point of contact is assumed to be on
$X$ - axis. A modified Riemann zeta function is proposed which is incorporated to the spherical coordinate system and real analysis has been performed. Through analytic continuation, the single point of contact of the droplet at the instant of touching the plane is analyzed. The zeta function is extracted at the point of collapse of the drop where it possesses a high probability to exhibit the property of a surge. Generally, continuous functions are a set of mathematical functions classically defined in a variety of ways [1]-[5]. They are seen to possess various useful properties which can be utilized in many applications including modeling. Surge waveforms have a lot of practical significance in many areas of mathematics and engineering [6]-[8]. A dying-surge can be defined as one whose amplitude reaches very high values (infinite) in almost zero (very short) time interval and then reduces to arbitrarily small values before settling down to zero. A dying-surge can also be defined in the spatial domain in a similar way. Functionally, this is entirely different from the common damped oscillations present in many electrical systems [9]-[10]. Surges are considered to be discontinuous functions and can be usually described using Dirac delta functions [11]-[12] or Kronecker delta functions [13]-[14]. But descriptions using Dirac delta functions possess many mathematical and analytical constraints. Surges are invariably present in all electrical and electronic circuits, and normal analysis cannot provide a comprehensive solution in real time scenarios. The analytical methodology proposed in this paper is presented in the following sections.

\section{RIEMANN ZETA FUNCTION MODELING OF A 2- MILLIMETER RADIUS FALLING WATER DROPLET}

Defining the Riemann zeta function as $\zeta(s)=\sum_{n=1}^{\infty} n^{-s}$ with $s$ being a complex variable with real part greater than unity. The water droplet is assumed to be freely falling just before the point of impact and spherical coordinate system is assigned for analysis. The total kinetic energy of the droplet of $2 \mathrm{~mm}$ radius, just before the point of impact, is considered. This finite kinetic energy of the droplet is modeled as Riemann zeta function of appropriate parameter $s$, with the converging infinite summation corresponding to the kinetic energies of individual molecules or molecular cluster level units. Since the droplet is assumed to be a perfect sphere in 
modeling, the first point of impact will be a tangential plane to the sphere.

For the droplet, just before the impact, analyzing kinetic energy as follows:

$\int_{\phi=0}^{2 \pi} \int_{\theta=0}^{\pi} \int_{r=0}^{\infty} f(r, \theta, \phi) r^{2} \sin \theta d r d \theta d \phi=\frac{1}{2} m v^{2}=$

$\frac{32}{3} \pi \times 10^{-7} v^{2}=\frac{64 \pi g d}{3} \times 10^{-7}$,

where $d$ is the distance through which the droplet has fallen through and $g$ is the acceleration due to gravity and $m$ being the mass of the droplet. With the RHS of (1) being finite, the integral evaluates to a finite value. Therefore, for the droplet, applying the spherical coordinates and defining the modified Riemann zeta function for the scenario under consideration will yield the following equations:

$$
\begin{aligned}
S_{\text {mod }}(r)=\sum_{n=1}^{\infty} n^{-s} & =\sum_{n=1}^{\infty} n^{-r \sin \theta(\cos \phi+j \sin \phi)} \\
& =\sum_{n=1}^{\infty} n^{-r^{\sin \theta(\cos \phi+j \sin \phi)}}
\end{aligned}
$$

With,$f(r, \theta, \phi)=\varsigma_{\text {mod }}(r)=\sum_{n=1}^{\infty} n^{-r \sin \theta(\cos \phi+j \sin \phi)}$

eqn (1) becomes,

$$
\begin{array}{r}
\int_{\phi=0}^{2 \pi} \int_{\theta=0}^{\pi} \int_{r=0}^{0.002} S_{\text {mod }}(r) r^{2} \sin \theta d r d \theta d \phi=\frac{1}{2} m v^{2} \\
=\frac{32}{3} \pi \times 10^{-7} v^{2}=\frac{64 \pi g d}{3} \times 10^{-7}
\end{array}
$$

Finiteness of the integral is ensured from eqn (3).

At the instant of collision of the water droplet with a horizontal $X-Y$ plane, the spherical coordinate system is assumed to be collapsed, with the initial point of impact defined on the $X$-axis in the Cartesian coordinate system. Therefore, the tangent which is the initial point of impact, can be obtained as the gradient of $f(r, \theta, \varphi)$ or $\varsigma_{\bmod }(r)$.

$$
\begin{aligned}
& \nabla \varsigma_{\text {mod }}(r)=\sum_{n=1}^{\infty} n^{-r \sin \theta \cos \phi} \ln \left(n^{-\sin \theta \cos \phi}\right) \hat{r} \\
& +\frac{1}{r} \sum_{n=1}^{\infty} n^{-r \sin \theta \cos \phi} \ln \left(n^{-r \cos \phi}\right) \cos \theta \hat{\theta} \\
& -\frac{1}{r \sin \theta} \sum_{n=1}^{\infty} n^{-r \sin \theta \cos \phi} \ln \left(n^{-r \sin \theta}\right) \sin \phi \hat{\phi}
\end{aligned}
$$

At this condition, i.e. at the point of collapse of spherical coordinate, assumed to be happening on the $X$-axis, $\varsigma_{\text {mod }}(r)=\sum_{n=1}^{\infty} n^{-x}$, and the derivative converges to a finite value only for certain conditions. In other cases when that does not happen, there exists the probability of existence of surges. These are very high valued- functions that are generated at the point of impact and in fact emerged from a water droplet of finite energy for the scenario under consideration. It is as if the infinitesimally small energy units, all trying to add up at the first single point of contact, resulting in a surge. This function, which will eventually die away with respect to space and time, can be used in the modeling of the underlying function.

\section{FUNCTIONAL ANALYSIS OF DYING-SURGES}

Let an arbitrary unknown continuous function be denoted by $y=f(x)$. Instead of the spatial domain $x$, the time domain $t$ can also be considered in the analysis. Defining the special property for this function as in eqn (5):

$$
a f^{\prime}(x)+b \int f(x) d x=0,
$$

where $f^{\prime}(x)$ denotes the first derivative of the function $f(x)$ and $a$ and $b$ are constants.

From eqn (5), the following condition can be obtained as:

$$
b f(x)=-a f^{\prime \prime}(x),
$$

where $f^{\prime \prime}(x)$ denotes the second derivative of $f(x)$. For defining such a real function at some finite points, let the variable $x$ be defined as $x=\left[x_{1}, x_{2}, x_{3} \ldots . x_{n}\right]$ and the corresponding function values as $y=\left[y_{1}, y_{2}, y_{3}, \ldots . . y_{n}\right]$. The points $x_{i}$ are defined close together such that:

$$
x_{i}=x_{i-1}+\varepsilon
$$

With this condition, evaluating the approximate first derivative of function $y=f(x)$ as in eqn (8):

$$
f^{\prime}(x)=\left[\frac{\left(y_{2}-y_{1}\right)}{\left(x_{2}-x_{1}\right)}, \frac{\left(y_{3}-y_{2}\right)}{\left(x_{3}-x_{2}\right)}, \ldots \ldots \ldots \ldots, \frac{\left(y_{n}-y_{n-1}\right)}{\left(x_{n}-x_{n-1}\right)}\right]
$$

with the condition that $x_{1}$ and $x_{2}$ very close together.

Evaluating the second derivative of $f(x)$ as follows:

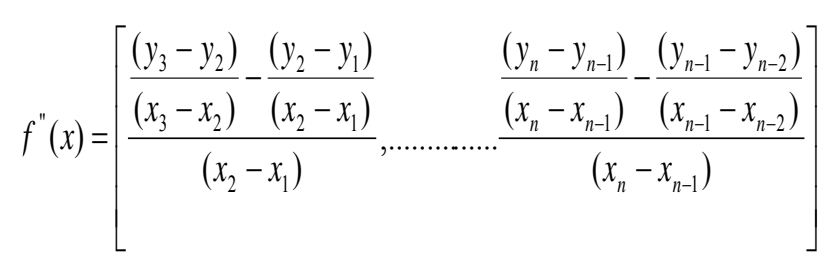

From the condition in eqn (6), with $a=b=1$, the following functions can be derived:

$$
y_{1}=-\left[\frac{\frac{\left(y_{3}-y_{2}\right)}{\left(x_{3}-x_{2}\right)}-\frac{\left(y_{2}-y_{1}\right)}{\left(x_{2}-x_{1}\right)}}{\left(x_{2}-x_{1}\right)}\right]
$$




$$
y_{2}=-\left[\frac{\frac{\left(y_{4}-y_{3}\right)}{\left(x_{4}-x_{3}\right)}-\frac{\left(y_{3}-y_{2}\right)}{\left(x_{3}-x_{2}\right)}}{\left(x_{3}-x_{2}\right)}\right] .
$$

Therefore, for the general case, this becomes:

$$
y_{i}=-\left[\frac{\frac{\left(y_{i+2}-y_{i+1}\right)}{\left(x_{i+2}-x_{i+1}\right)}-\frac{\left(y_{i+1}-y_{i}\right)}{\left(x_{i+1}-x_{i}\right)}}{\left(x_{i+1}-x_{i}\right)}\right]
$$

Solving for $y_{i}$ gives the following equation:

$$
y_{i}=\left[\frac{\left(-x_{i+1} y_{i+2}-x_{i} y_{i+1}+x_{i+2} y_{i+1}\right)}{\left(\left(x_{i+1}-x_{i}\right)^{2}\left(x_{i+2}-x_{i+1}\right)\right)-\left(x_{i+2}-x_{i+1}\right)}\right]
$$

\section{RESULTS: REVERSE SYNTHESIS OF THE PROPOSED}

\section{FUNCTION}

From eqn (11), the proposed function can be reversesynthesized for any arbitrary point. The function value $y_{i}$ can be synthesized using $x_{i}, x_{i+1}, x_{i+2}, y_{i+1}$ and $y_{i+2}$. In the proposed method, the function is reverse synthesized with two values $y_{i+1}$ and $y_{i+2}$, with the final value arbitrarily close to zero. Synthesis is carried out using the simulation platform $M_{A T L A B}{ }^{\circledR}$ for 100,1000 and 10000 values of the function with closely spaced values of $x$. Figures 1 through 3 depict the reverse synthesized functions. Table 1 shows the synthesized function values. Initial 10 values for $n=10$ synthesis are shown while the first 180 values for $n=1000$ synthesis and first 9180 values for $n=10000$ synthesis tend to infinitely large.

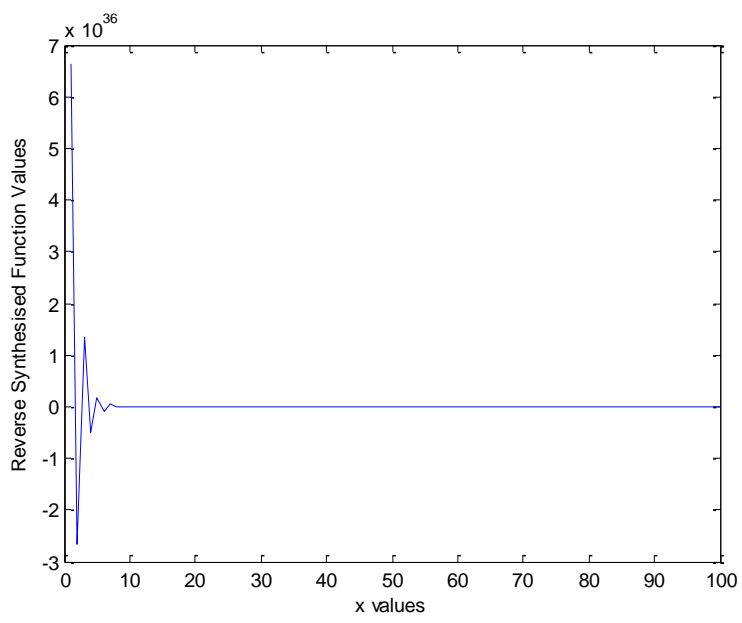

Fig 1.Reverse synthesis for 100 values- first 10 function values possessing very large magnitudes

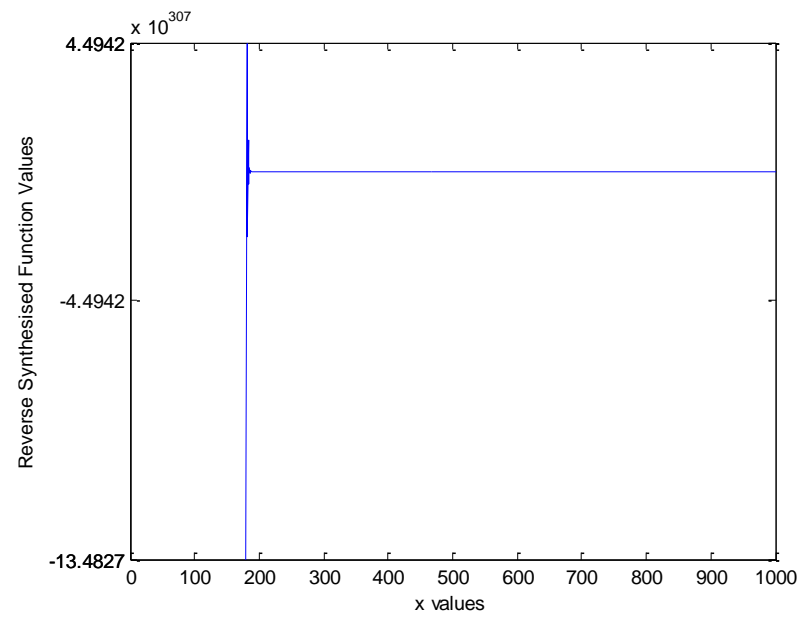

Fig 2. Reverse synthesis for 1000 values- first 180 values are infinitely large

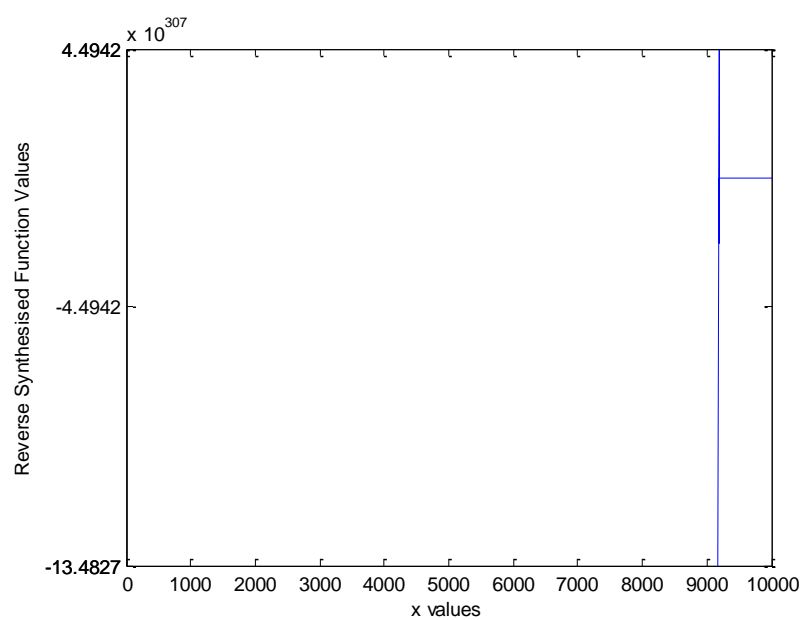

Fig 3 . Reverse Synthesis for 10000 values- first 9180 values are infinitely large

Table 1: Synthesized function values -proposed function for $n$ point reverse synthesis $(n=100,1000,10000)$.

\begin{tabular}{|l|l|l|}
\hline $\begin{array}{c}\text { First 10 values }(\mathbf{1 0 0} \\
\text { point synthesis) }\end{array}$ & $\begin{array}{c}\mathbf{1 8 1}^{\text {th }} \text { to 190 } \\
\text { values(1000-point } \\
\text { synthesis) }\end{array}$ & $\begin{array}{c}\mathbf{9 1 8 1}^{\text {th }} \text { to } 9190^{\text {th }} \\
\text { values }(\mathbf{1 0 0 0 0 -} \\
\text { point synthesis })\end{array}$ \\
\hline $6.646 \times 10^{36}$ & $1.348 \times 10^{308}$ & $0.449 \times 10^{308}$ \\
\hline$-2.658 \times 10^{36}$ & $-0.674 \times 10^{308}$ & $-0.224 \times 10^{308}$ \\
\hline $1.329 \times 10^{36}$ & $0.224 \times 10^{308}$ & $0.112 \times 10^{308}$ \\
\hline$-0.498 \times 10^{36}$ & $-0.112 \times 10^{308}$ & $-0.042 \times 10^{308}$ \\
\hline $0.1662 \times 10^{36}$ & $0.0421 \times 10^{308}$ & $0.014 \times 10^{308}$ \\
\hline$-0.083 \times 10^{36}$ & $-0.014 \times 10^{308}$ & $-0.007 \times 10^{308}$ \\
\hline $0.041 \times 10^{36}$ & $0.010 \times 10^{308}$ & $0.003 \times 10^{308}$ \\
\hline$-0.015 \times 10^{36}$ & $-0.004 \times 10^{308}$ & $-0.001 \times 10^{308}$ \\
\hline $0.0052 \times 10^{36}$ & $0.001 \times 10^{308}$ & $0.0004 \times 10^{308}$ \\
\hline$-0.0026 \times 10^{36}$ & $-0.0007 \times 10^{308}$ & $-0.0002 \times 10^{308}$ \\
\hline
\end{tabular}

From Table 1, initial 10 values for synthesis with $n=10$ is shown while the first 180 values for $n=1000$ synthesis and first 9180 values for $n=10000$ synthesis tend to infinitely large.

From the proposed synthesized function, it can be seen that the initial values all attain very high magnitudes which can be fairly assumed to be close to infinity in a practical scenario. Additionally, from Table 1, the noticeably finite set of values tend to have very high magnitudes. The synthesis is done by arbitrarily-assumed final and penultimate values of 0.005 and 
1 respectively. The final value is chosen to be close to zero where the surge has died out. From the plots generated for the synthesized function and the tabulated values, it can be seen that the proposed function corresponds to a dying-surge. Therefore, the dying- surge is modeled as a continuous function as per eqn (5).

Since the sinusoid or the cosinusoid i.e. $y=\sin x$ and $y=\cos x$ satisfy the condition in eqn (5), the above Dyingsurge model also corresponds to a sine or cosine function.

\section{A. General Case Analysis}

A special case of eqn (5) can be written as in eqn (12):

$$
f(x)=-f^{\prime \prime}(x)+g(x)
$$

where $g(x)$ is a general function. Any function $f(x)$ that can be represented as in eqn (12), can be modeled using the proposed methodology. With reference to eqn (11), for this general case, it can be shown that

$$
y_{i}=\left[\frac{\left(-x_{i+1} y_{i+2}-x_{i} y_{i+1}+x_{i+2} y_{i+1}\right)+g(x)\left(x_{i+1}-x_{i}\right)^{2}\left(x_{i+2}-x_{i+1}\right)}{\left(\left(x_{i+1}-x_{i}\right)^{2}\left(x_{i+2}-x_{i+1}\right)\right)-\left(x_{i+2}-x_{i+1}\right)}\right]
$$

Since the points $x_{i}$ are required to be very close together, eqn (13) will also correspond to the Dying -surge model as demonstrated in the following example:

\section{Case: Arbitrary Function:}

Let a general function be defined as: $g(x)=1+\frac{x^{2}}{2}$

This corresponds to the function $f(x)=x$. The reverse synthesized Dying -surge model of the function can be simulated as in Fig 4.

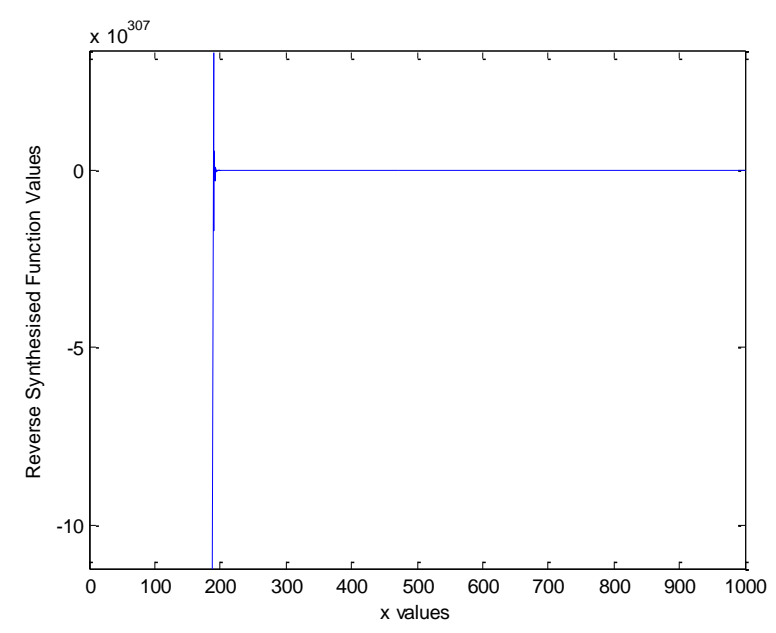

Fig 4. Dying -surge model for the function $y=x$

The Dying- surge Model corresponds to an electrical squeezing of waveform, existent over infinite values, combined to a finite number of values placed very close together, with defined final and penultimate values. This 'squeezing' results in the surge which will die out as it proceeds from start to the pre-defined final value, close to zero. Therefore, any function which is finite, continuous valued, integrable and differentiable can be expressed as a dying- surge over an arbitrarily finite number of points.

\section{B. Applications}

In physical scenarios like the case of a falling water droplet and also in electrical and electronic circuits, dying-surges occur at different spatial points and time instants, due to many unknown reasons. The surges can in many cases, be measured, and from the sampled finite values, the underlying function producing the dying-surge can be estimated.

\section{CONCLUSION}

The Riemann zeta associated with the finite energy possessed by a $2 \mathrm{~mm}$ radius, free falling water droplet impinging on a plane is analyzed. A modified zeta function is proposed which is defined in the spherical coordinates and real analysis has been performed. Making use of real analytic continuation, the single point of contact of the drop at the instant of touching the plane on $X$ - axis is analyzed. The zeta function is extracted at the point of collision of the droplet where it possesses a probability to exhibit the property of a surge. Continuous functions, where the function's first derivative and first integral add together to a nullity at all points, can be reverse synthesized, resulting in the dying-surge waveform. The proposed concept can be extended to general continuous real functions. This corresponds to the synthesis of the function's Dying-surge model. The Dying- surge model represents an electrical squeezing or compression of a waveform and can be helpful in the estimation of the unknown underlying function of a surge.

\section{ACKNOWLEDGMENT}

The author gratefully acknowledges the support provided by Govt. Model Engineering College, Ernakulam for carrying out this work.

Conflicts of Interest: There are no conflicts of interest to declare

\section{REFERENCES}

[1]. Harper, J.F, "Defining continuity of real functions of real variables", BSHM Bulletin: Journal of the British Society for the History of Mathematics ,2016, pp. 1-16.

[2]. Rusnock, P, Kerr-Lawson A., "Bolzano and uniform continuity", Historia Mathematica, vol.32 , no.3, 2005, pp. 303-311.

[3]. Goursat, E., A course in mathematical analysis, Boston: Ginn, USA, 1904.

[4]. Hewitt Edwin, Stromberg Karl, Real and Abstract analysis, Springer-Verlag, New York, 1965

[5]. Rudin, Walter, Real and complex analysis, McGraw-Hill, New York, 1965

[6]. S. Okabe, S. Yuasa and S. Kaneko, "Evaluation of breakdown characteristics of gas insulated switchgears for non-standard lightning impulse waveforms - breakdown characteristics for non-standard lightning impulse waveforms associated with disconnector switching surges", IEEE Transactions on Dielectrics and Electrical Insulation, vol.15, no. 3, 2008 , pp.721-729.

[7]. R. Qi, B. Li and Y. Du, "Induced surges in railway signaling systems during an indirect lightning strike", 
Proc. 33rd International Conference on Lightning Protection (ICLP), Estoril, Portugal, 2016, pp.1-5.

[8]. D. Miller, R. Kennel, M. Reddig and M. Schlenk, "Surge immunity test analysis for modern switching mode power supplies", Proc. IEEE International Telecommunications Energy Conference (INTELEC), Austin, Texas, 2016 , pp.1-6.

[9]. V. Arcidiacono, E. Ferrari and F. Saccomanno, "Studies on damping of electromechanical oscillations in multimachine systems with longitudinal structure", IEEE Transactions on Power Apparatus and Systems , vol. 95 , no.2, 1976, pp.450-460.

[10].D. Robertson, "A mode of studying damped oscillations by the aid of shrinking vectors", Journal of the Institution in Electrical Engineers, vol.54, no.251, 1915, pp.24-34.

[11]. R. Bracewell, The Fourier Transform and its applications, 2nd ed., McGraw- Hill, New York, 1986.

[12].J. Shen and A. Kost, "Modelling of the idealized exciting current sources in the FEM", IEEE Transactions on Magnetics, vol.31, no.3, 1995, pp.1368-1371.

[13]. Eugene Spiegel and Christopher J. O'Donnell, Incidence Algebras, Marcel Dekker, New York, 1997.

[14]. Wilfred Kaplan, Advanced Calculus, 5th ed., AddisonWesley, California, 2002.

\section{AUTHOR PROFILE}

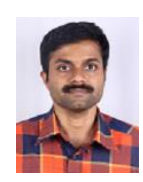
Govt. Model Engineering College-APJ Abdul Kalam Technological University, Ernakulam, India, as associate professor in the department of electronics engineering. He has acquired his $\mathrm{PhD}$ in Engineering from Cochin University of Science and Technology and Masters degree from Anna University, Chennai. He has to his credit, many international journal and conference publications and an active researcher in the areas of signal and image processing, applied mathematics and optics.

\section{Creative Commons Attribution License 4.0 (Attribution 4.0 International, CC BY 4.0)}

This article is published under the terms of the Creative Commons Attribution License 4.0 https://creativecommons.org/licenses/by/4.0/deed.en US 